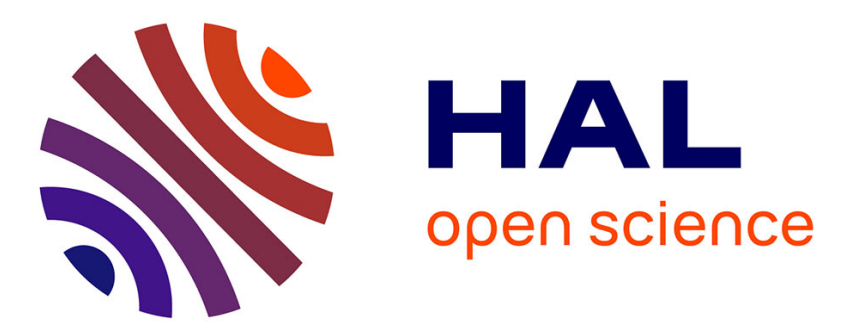

\title{
A rescaled elliptic relaxation approach: Neutralizing the effect on the log layer
}

\author{
Remi Manceau, J. R Carlson, T. Gatski
}

\section{To cite this version:}

Remi Manceau, J. R Carlson, T. Gatski. A rescaled elliptic relaxation approach: Neutralizing the effect on the $\log$ layer. Physics of Fluids, 2002, 14 (11), pp.3868-3879. 10.1063/1.1511547 . hal02990484

\section{HAL Id: hal-02990484 \\ https://hal.science/hal-02990484}

Submitted on 5 Nov 2020

HAL is a multi-disciplinary open access archive for the deposit and dissemination of scientific research documents, whether they are published or not. The documents may come from teaching and research institutions in France or abroad, or from public or private research centers.
L'archive ouverte pluridisciplinaire HAL, est destinée au dépôt et à la diffusion de documents scientifiques de niveau recherche, publiés ou non, émanant des établissements d'enseignement et de recherche français ou étrangers, des laboratoires publics ou privés. 


\title{
A rescaled elliptic relaxation approach: Neutralizing the effect on the log layer
}

\author{
R. Manceau ${ }^{\text {a) }}$ \\ Laboratoire d'Études Aérodynamiques, UMR CNRS 6609, université de Poitiers, SP2MI, Téléport 2, \\ Bd Marie et Pierre Curie, BP 30179, 86962 Futuroscope Chasseneuil, France \\ J. R. Carlson and T. B. Gatski \\ Computational Modeling \& Simulation Branch, NASA Langley Research Center, Hampton, Virginia 23681
}

(Received 21 November 2001; accepted 6 August 2002; published 18 September 2002)

\begin{abstract}
An alternative scaling for the relaxation function describing the velocity pressure-gradient correlation used in the elliptic relaxation procedure for both eddy-viscosity and Reynolds stress models is presented. While other alternatives have been proposed to neutralize the adverse effect on log-layer dynamics, they have relied on altering the original differential formulation. A simpler alternative is presented here that involves a rescaling of the relaxation function with the isotropic dissipation rate as well as the turbulent kinetic energy. Various comparative tests are made and the new rescaled formulation is shown to provide improved and accurate predictions for both the eddy-viscosity and Reynolds stress models. (C) 2002 American Institute of Physics.
\end{abstract}

[DOI: $10.1063 / 1.1511547$ ]

\section{INTRODUCTION}

Recent studies ${ }^{1,2}$ utilizing the elliptic relaxation approach, proposed by Durbin ${ }^{3,4}$ for accurate near-wall eddyviscosity and Reynolds stress modeling have shown that, despite the overall benefit, the formulation negatively impacts the log-layer predictions of such models. Manceau et al. ${ }^{5}$ have shown from a DNS database analysis that the cause can be attributed to the isotropy assumption for the two-point correlation function used in the derivation of the elliptic relaxation equation. Modifying the model to better replicate the underlying physics has led to alternate forms for the elliptic relaxation equation that neutralize the impact on the logarithmic layer. However, these new formulations involve additional differential operators, which, when coupled with a full Reynolds stress formulation, may affect the computational robustness of the system in more complex flow fields.

An alternative, isotropic rescaling of the tensor function representing the redistribution term in the turbulent Reynolds stress equations is proposed. The rescaling now involves the isotropic dissipation rate as well as the turbulent kinetic energy that has been used in all previous elliptic relaxation formulations. While the inclusion of the isotropic dissipation rate in the scaling has little or no impact on the redistribution in the near-wall region, it is found to remove its amplification in the log-layer region when only the turbulent kinetic energy is used in the scaling.

Several comparisons are made with both direct numerical simulations of channel flow ${ }^{6}$ at values of friction velocity Reynolds number $\operatorname{Re}_{\tau}\left(=u_{\tau} h / \nu, u_{\tau}\right.$ friction velocity, and $h$ channel half-width) ranging from 180 to 590, and experimental data ${ }^{7,8}$ at $\operatorname{Re}_{\tau}=1017$. For these channel flows both the $\overline{v^{2}}-f$ and Reynolds stress elliptic relaxation approaches are

${ }^{a)}$ Electronic mail: remi.manceau@lea.univ-poitiers.fr evaluated using results for both mean and turbulent quantities. In order to further validate the new formulation, the rescaled $\overline{v^{2}}-f$ model is applied to the case of turbulent boundary layer flow. Comparisons with $\mathrm{DNS}^{9}$ and experimental data ${ }^{10}$ of skin-friction variation with Reynolds number and mean velocity predictions further highlight the benefits of the rescaled elliptic relaxation formulation.

\section{ORIGINAL AND RESCALED ELLIPTIC RELAXATION FORMULATIONS}

It is useful at the outset to outline the methodology used in the previous elliptic relaxation formulations. Initially, ${ }^{3}$ the elliptic relaxation equation had been written as

$$
f_{i j}-L^{2} \nabla^{2} f_{i j}=f_{i j}^{h},
$$

where $f_{i j}$ was defined by

$$
f_{i j}=\frac{1}{K}\left(-\overline{u_{i} \frac{\partial p}{\partial x_{j}}}-\overline{u_{j} \frac{\partial p}{\partial x_{i}}}-\varepsilon_{i j}+\varepsilon_{i j}^{*}\right)=\frac{1}{K}\left[\phi_{i j}-\varepsilon_{i j}+\varepsilon_{i j}^{*}\right]
$$

and

$$
f_{i j}^{h}=\frac{1}{K}\left[\Pi_{i j}-\frac{2}{3} \varepsilon \delta_{i j}+\varepsilon_{i j}^{*}\right],
$$

with $\varepsilon_{i j}^{*}$ the anisotropic form of the dissipation rate in the vicinity of the wall, and $\Pi_{i j}$ a high-Reynolds number pressure-strain rate correlation model. Different (high Reynolds number) pressure-strain rate models can be used. For example, Wizman et al. ${ }^{1}$ compared the results in a channel flow using three different models for $\Pi_{i j}$ : Launder, Reece, and Rodi, ${ }^{11}$ Craft and Launder, ${ }^{12}$ and Speziale, Sarkar, and Gatski $^{13}$ (SSG). They have shown that since $\varepsilon$ and (any) $\Pi_{i j}$ model behave as $y^{-1}$ along with $K$ constant in the logarith- 

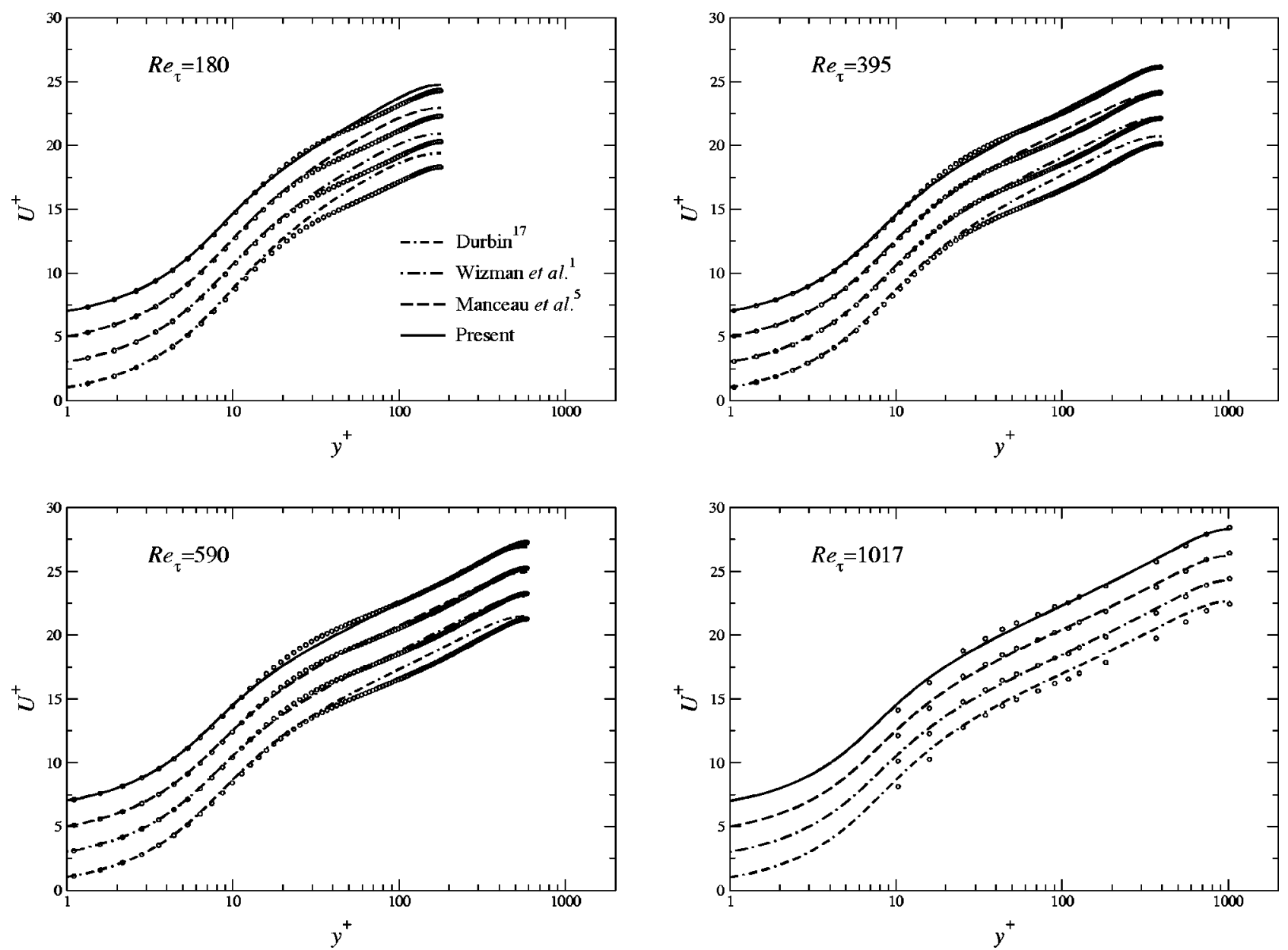

FIG. 1. A comparison of mean velocity profiles from $\overline{v^{2}}-f$ models with DNS (Ref. 6) and experimental data (Refs. 7, 8) in channel flow.

mic layer, the solution of Eq. (1) is $f_{i j}=\Gamma f_{i j}^{h}$ where $\Gamma$ is the amplification factor, with $\Gamma=1.51$. Manceau et al. ${ }^{5}$ have summarized the size of this amplification factor for different formulations of the elliptic relaxation equation.

As shown by Manceau, ${ }^{14}$ Eq. (1) is a consequence of the modeling of $\Psi_{i j}\left(\mathbf{x}, \mathbf{x}^{\prime}\right)$, the two-point correlation between the fluctuating velocity and the Laplacian of the pressure gradient. Indeed, $\Psi_{i j}\left(\mathbf{x}, \mathbf{x}^{\prime}\right)$ appears in the integral equation of $\phi_{i j}$ :

$$
\rho \phi_{i j}=-\int_{\Omega} \frac{\Psi_{i j}\left(\mathbf{x}, \mathbf{x}^{\prime}\right)}{4 \pi\left\|\mathbf{x}^{\prime}-\mathbf{x}\right\|} \mathrm{dV}\left(\mathbf{x}^{\prime}\right)
$$

If $\Psi_{i j}\left(\mathbf{x}, \mathbf{x}^{\prime}\right)$ is modeled by

$$
\frac{\Psi_{i j}\left(\mathbf{x}, \mathbf{x}^{\prime}\right)}{K(\mathbf{x})}=\frac{\Psi_{i j}\left(\mathbf{x}^{\prime}, \mathbf{x}^{\prime}\right)}{K\left(\mathbf{x}^{\prime}\right)} \exp \left(-\frac{r}{L}\right)
$$

(where the scaling by $K$ is needed for preserving the correct limiting behavior of $\phi_{i j}$ in the near-wall region), Eq. (4) can be inverted to give Eq. (1). However, Manceau et al. ${ }^{5}$ showed that using an isotropic exponential correlation function leads to overweighting the points $\mathbf{x}^{\prime}$ in the region between the current point $\mathbf{x}$ and the wall in Eq. (4). Since $\Psi_{i j}\left(\mathbf{x}^{\prime}, \mathbf{x}^{\prime}\right)$ decreases like $1 / y^{\prime}$ in the log layer ( $K$ being con- stant), overweighting the region $y^{\prime}<y$ leads to overestimating $\phi_{i j}$ (this is the negative impact of the elliptic relaxation strategy quoted above).

Different formulations of the elliptic relaxation equation were proposed by Wizman et al., ${ }^{1}$ Laurence and Durbin, ${ }^{15}$ and Manceau et al. ${ }^{5}$ in order to avoid this undesired effect. All of these alternatives were based on modifying the elliptic operator $\left(1-L^{2} \nabla^{2}\right)$ and have an amplification factor close to or less than unity. ${ }^{5}$ In this study, two representative formulations are selected for comparison with the new rescaled formulation proposed here. These are the Wizman et al. ${ }^{1}$ neutral $(\Gamma=1)$ proposal,

$$
f_{i j}-\nabla^{2}\left(L^{2} f_{i j}\right)=f_{i j}^{h},
$$

and the Manceau et al. ${ }^{5}$ proposal,

$$
\left[1+16 \beta(\boldsymbol{\nabla} L)^{2}\right] f_{i j}-L^{2} \nabla^{2} f_{i j}-8 \beta L \nabla L \cdot \nabla f_{i j}=f_{i j}^{h},
$$

which is based on an anisotropic correlation function, and where $\Gamma$ is a function of $\beta .^{5}$ This functional dependence in the Manceau et al. ${ }^{5}$ model allows the adaptation of $\Gamma$ to the model used for $\Pi_{i j}$. For a model that overestimates the redistribution in the log layer, such as the Launder, Reece, and Rodi ${ }^{11}$ model used here for the $\overline{v^{2}}-f$ formulation, $\Gamma<1$ is preferable; however, for a model that gives a correct level of 

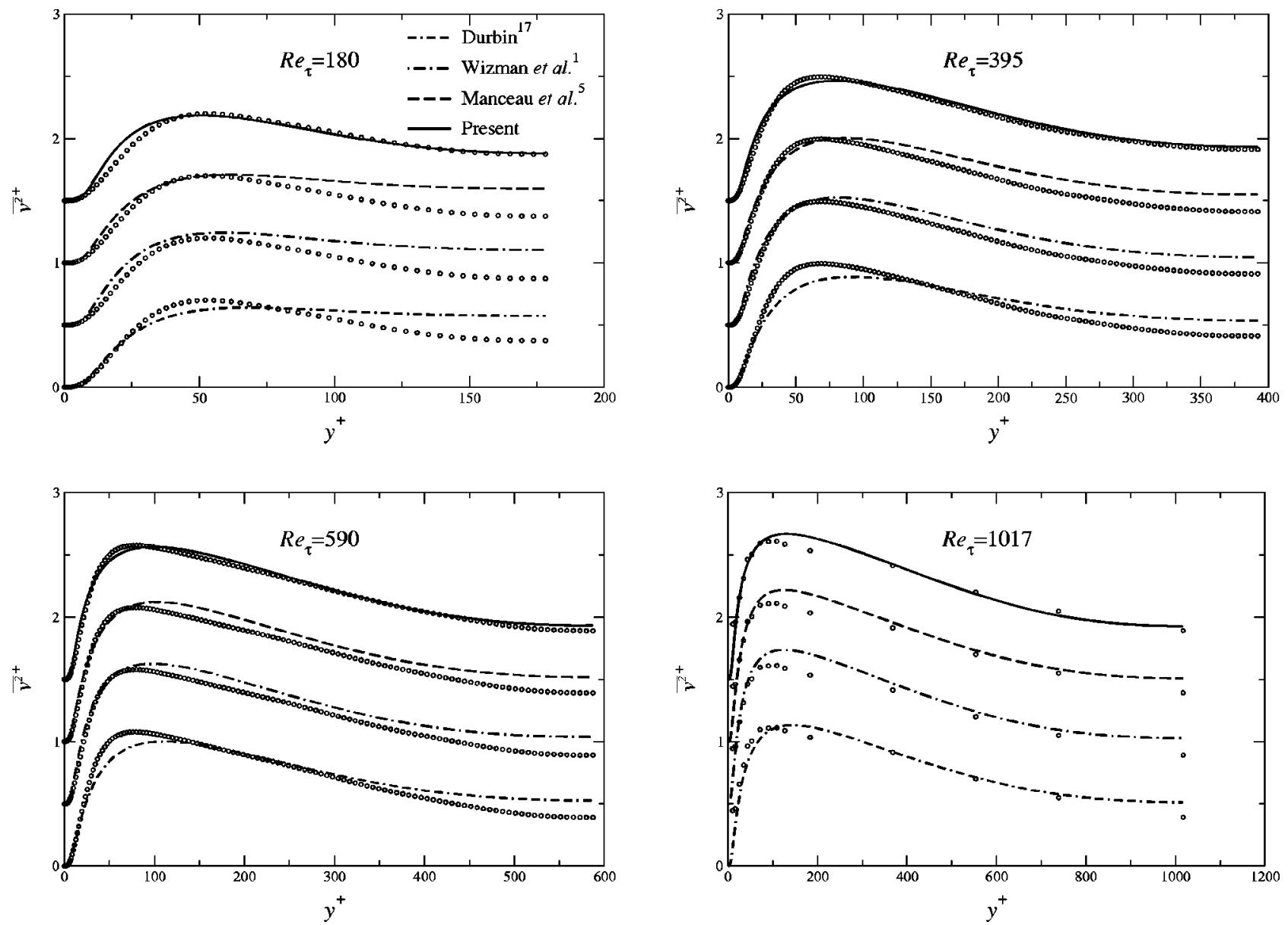

FIG. 2. A comparison of the $\overline{v^{2}+}$ turbulent normal stress component from $\overline{v^{2}}-f$ models with DNS (Ref. 6) and experimental data (Refs. 7, 8) in channel flow.

redistribution in the log layer, such as the $\mathrm{SSG}^{13}$ model used here for the Reynolds stress formulation, $\Gamma=1$ can be chosen. In this study, $\beta=0.12$ is used for the $\overline{v^{2}}-f$ formulation, leading to $\Gamma=0.7$, and $\beta=\frac{1}{12}$ for the Reynolds stress formulation, leading to $\Gamma=1$.

In contrast to earlier alternative formulations, the new formulation presented here is not based on the modification of the elliptic operator, but on a modified scaling in Eq. (5). In addition to the usual scaling by the turbulent kinetic energy $K$, the two-point correlation function is now also scaled by the isotropic dissipation rate $\varepsilon$. In the log layer, the Reynolds stresses and turbulent anisotropies are assumed constant and $\varepsilon(=\mathcal{P})$ is $\mathcal{O}\left(y^{-1}\right)$. As pointed out by Wizman et al., ${ }^{1}$ the redistribution term is also $\mathcal{O}\left(y^{-1}\right)$ and the new scaling $\varepsilon K$ negates the $y^{-1}$ behavior such that $f_{i j} \sim$ const in the log layer.

The dissipation rate scaling suppresses the effect of the overweighting due to the isotropy of the exponential function since $\Psi_{i j}\left(\mathbf{x}^{\prime}, \mathbf{x}^{\prime}\right) / K\left(\mathbf{x}^{\prime}\right) \varepsilon\left(\mathbf{x}^{\prime}\right)$ is constant in the log layer, and giving more weight to one side and less to the other has no consequence. Although introducing $\varepsilon$ into Eq. (5) can appear rather arbitrary, it is worth noting that $\varepsilon$ is a natural scale for $\phi_{i j}$ and, accordingly, for $\Psi_{i j}$. Therefore, the exponential shape assumption for the correlation function is at least as justified with the new scaling as with the former one.

The addition of the dissipation rate scaling directly af- fects the behavior of the elliptic relaxation equation in the logarithmic layer, so that $f_{i j}=f_{i j}^{h}(\Gamma=1)$. Equations (2) and (3) are now rewritten as

$$
f_{i j}=\frac{1}{\varepsilon K}\left[\phi_{i j}-\varepsilon_{i j}+\varepsilon_{i j}^{*}\right]
$$

and

$$
f_{i j}^{h}=\frac{1}{\varepsilon K}\left(\Pi_{i j}-\frac{2}{3} \varepsilon \delta_{i j}+\varepsilon_{i j}^{*}\right),
$$

respectively. Note that, similar to the original formulation, ${ }^{3}$ to avoid the occurrence of a singularity of the $f_{i j}$ equation at the wall, $K$ is bounded by $\varepsilon T_{\mathrm{K}}$, where $T_{\mathrm{K}}$ is the Kolmogorov time scale. Since in the vicinity of the wall $\varepsilon$ takes a nonzero value, the original (single scaling with $K$ ) limiting behavior of $f_{i j}$ is retained as soon as the wall boundary conditions for the different components of $f_{i j}$ are rescaled, that is

$$
\begin{aligned}
& f_{22}^{w}=-\frac{20 \nu^{2} \overline{v^{2}}}{\varepsilon^{2} y^{4}}, \quad f_{12}^{w}=-\frac{20 \nu^{2} \overline{u v}}{\varepsilon^{2} y^{4}}, \\
& f_{11}^{w}=f_{33}^{w}=-\frac{1}{2} f_{22}^{w},
\end{aligned}
$$

where $y$ is the distance normal to the wall. 

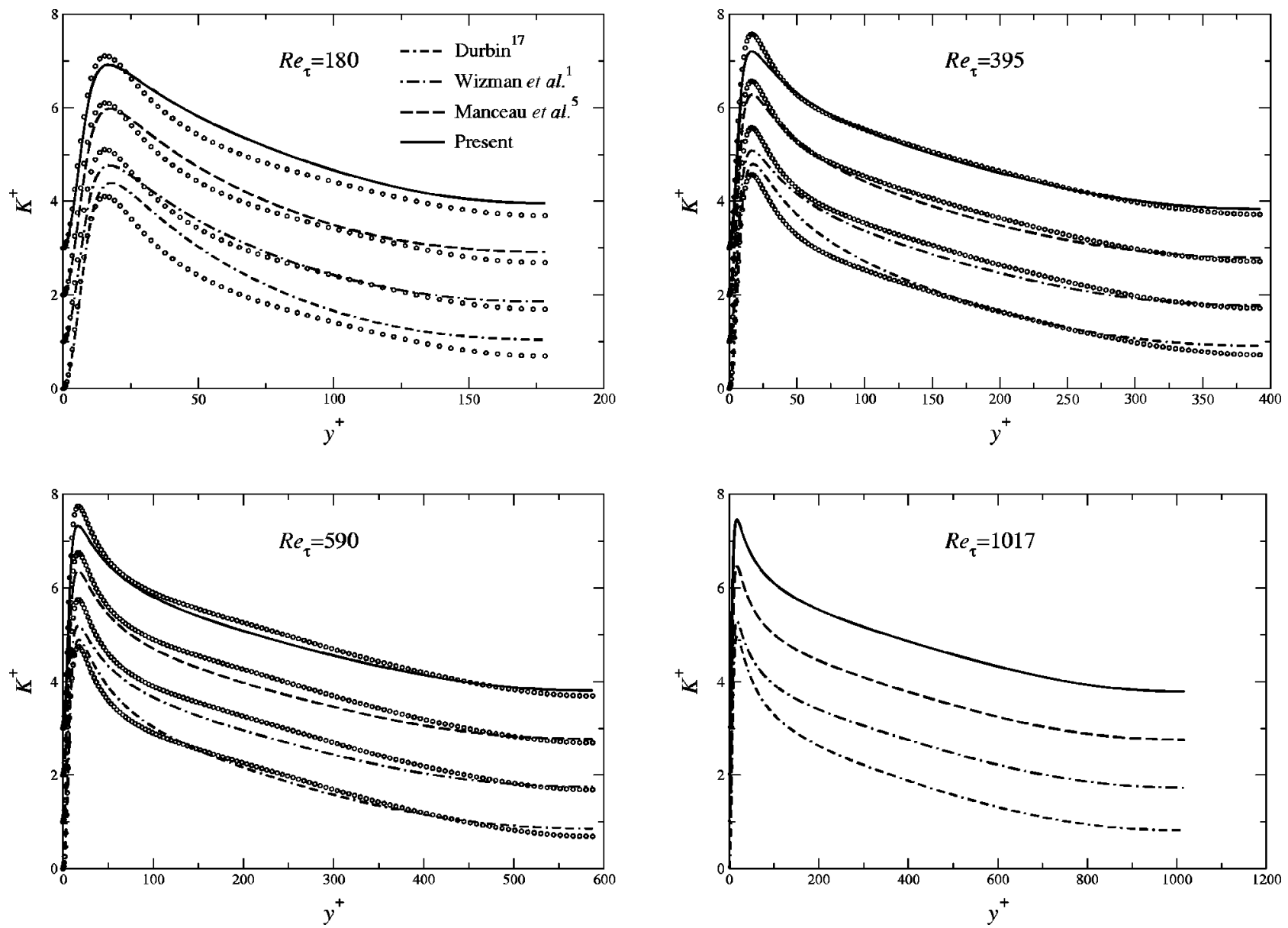

FIG. 3. A comparison of turbulent kinetic energy profiles from $\overline{v^{2}}-f$ models with DNS (Ref. 6) and experimental data (Refs. 7, 8) in channel flow.

Note that Durbin and Laurence ${ }^{16}$ proposed a similar rescaling, $f_{i j}=L \phi_{i j} / K$. Since $L$ behaves as $y$ in the logarithmic layer, this rescaling also neutralizes the effect of the elliptic operator in this region. However, since the turbulent length scale $K^{3 / 2} / \varepsilon$ goes to zero at the wall, this formulation changes the near-wall behavior of the model, unless the

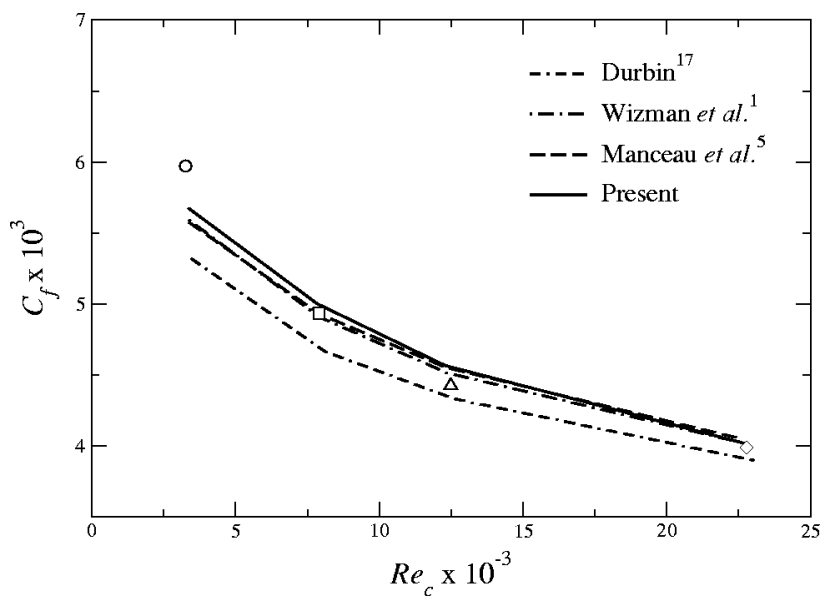

FIG. 4. Variation of a skin friction coefficient over Reynolds number range for $\overline{v^{2}}-f$ models in channel flow: $O, \operatorname{Re}_{c}=3260\left(\operatorname{Re}_{\tau}=180\right) ; \square, \operatorname{Re}_{c}$ $=7897\left(\operatorname{Re}_{\tau}=395\right) ; \triangle, \operatorname{Re}_{c}=12485\left(\operatorname{Re}_{\tau}=590\right) ; \diamond, \operatorname{Re}_{c}=22776\left(\operatorname{Re}_{\tau}\right.$ $=1017)$. length scale $L$ used in the normalization of $f_{i j}$ reverts to the Kolmogorov length scale in the near-wall region. From a computational standpoint, such changes in scaling definitions can be problematic if not done with care; otherwise, discontinuities can be introduced that can be further amplified when in any differentiation process. Such problems do not arise when the rescaling by $\varepsilon K$ is used.

The $\overline{v^{2}}-f$ model $^{3}$ is the reduction of the elliptic relax-

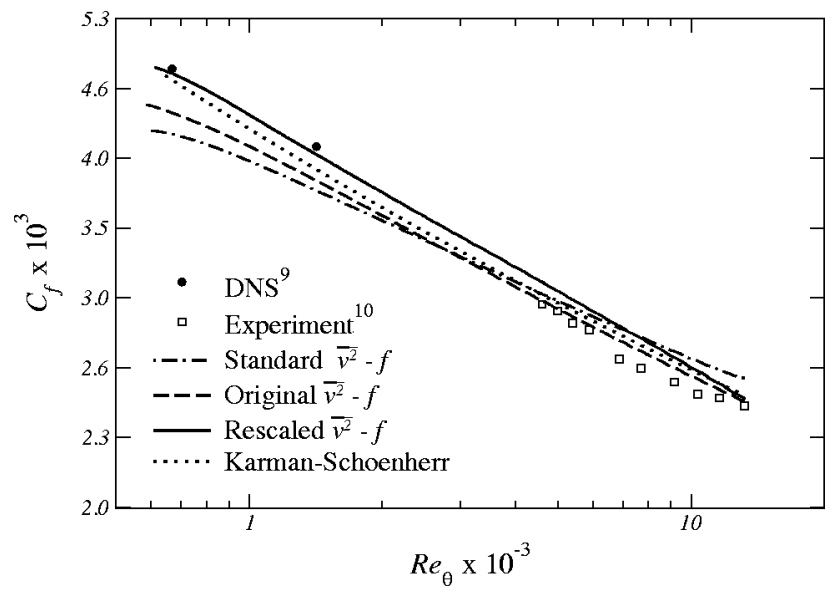

FIG. 5. Skin friction coefficient variation with a momentum thickness Reynolds number for $\overline{v^{2}}-f$ models (log-log scale) in boundary-layer flow. 

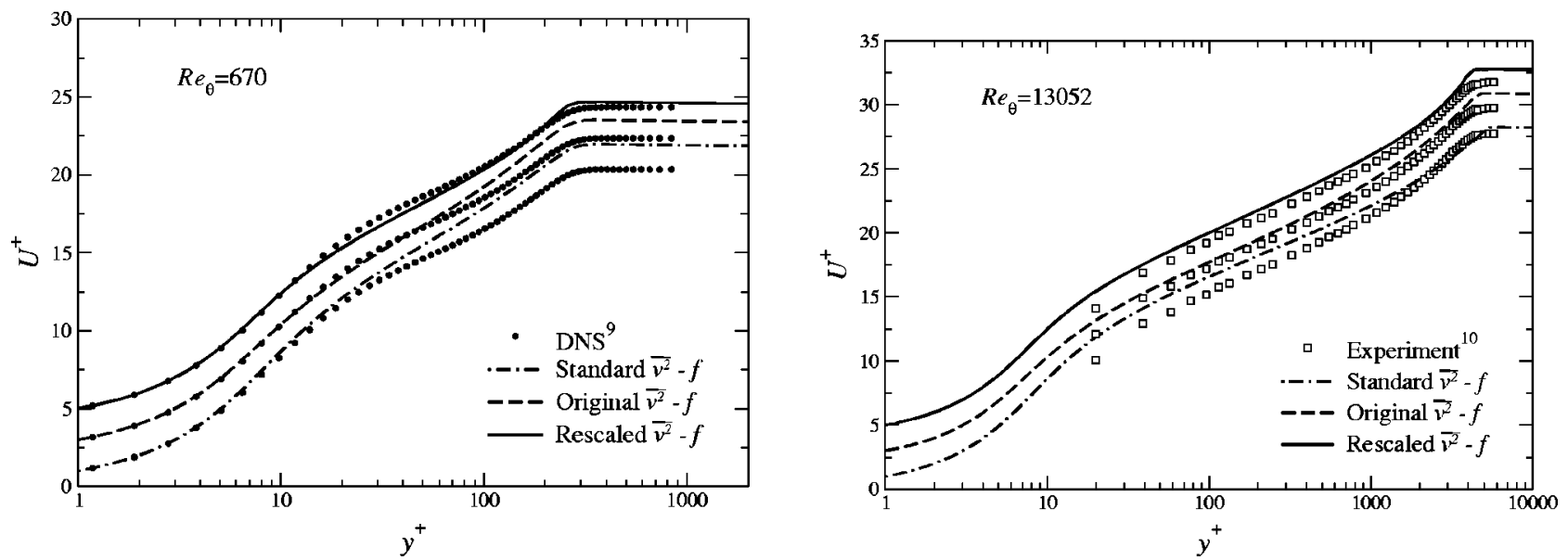

FIG. 6. A comparison of mean velocity profiles from $\overline{v^{2}}-f$ models with DNS (Ref. 9) and experimental data (Ref. 10) in boundary-layer flow.

ation procedure to three transport equations. The Reynolds stresses are obtained from the Boussinesq equation, with a modified eddy viscosity,

$$
\nu_{t}=C_{\mu} \overline{v^{2}} T,
$$

where $T$ is $K / \varepsilon$ but bounded near the wall by the Kolmogorov time scale $\sqrt{\nu / \varepsilon}$. The use of $\overline{v^{2}}$ instead of the standard
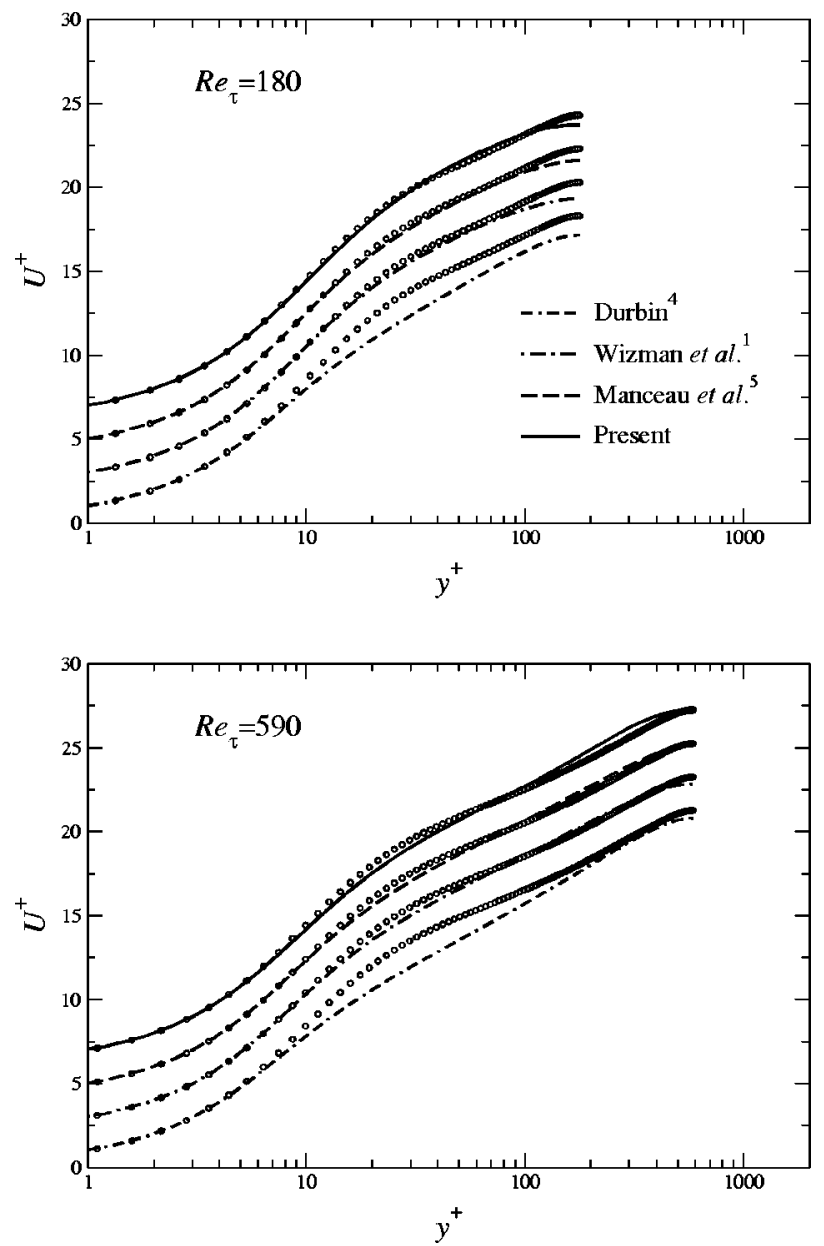

$K$ in (11) accounts for the blocking effect of the wall on turbulence diffusion without using empirical damping functions. A transport equation is solved for $\overline{v^{2}}$, directly taken from the full Reynolds stress model. Here $\overline{v^{2}}$ is considered an energy scale generalizing the wall-normal Reynolds stress component everywhere in the domain-a hypothesis that has proved successful in many flows. ${ }^{17-19}$ In addition, the anisotropic form of the dissipation rate tensor $\varepsilon_{i j}^{*}$ is taken
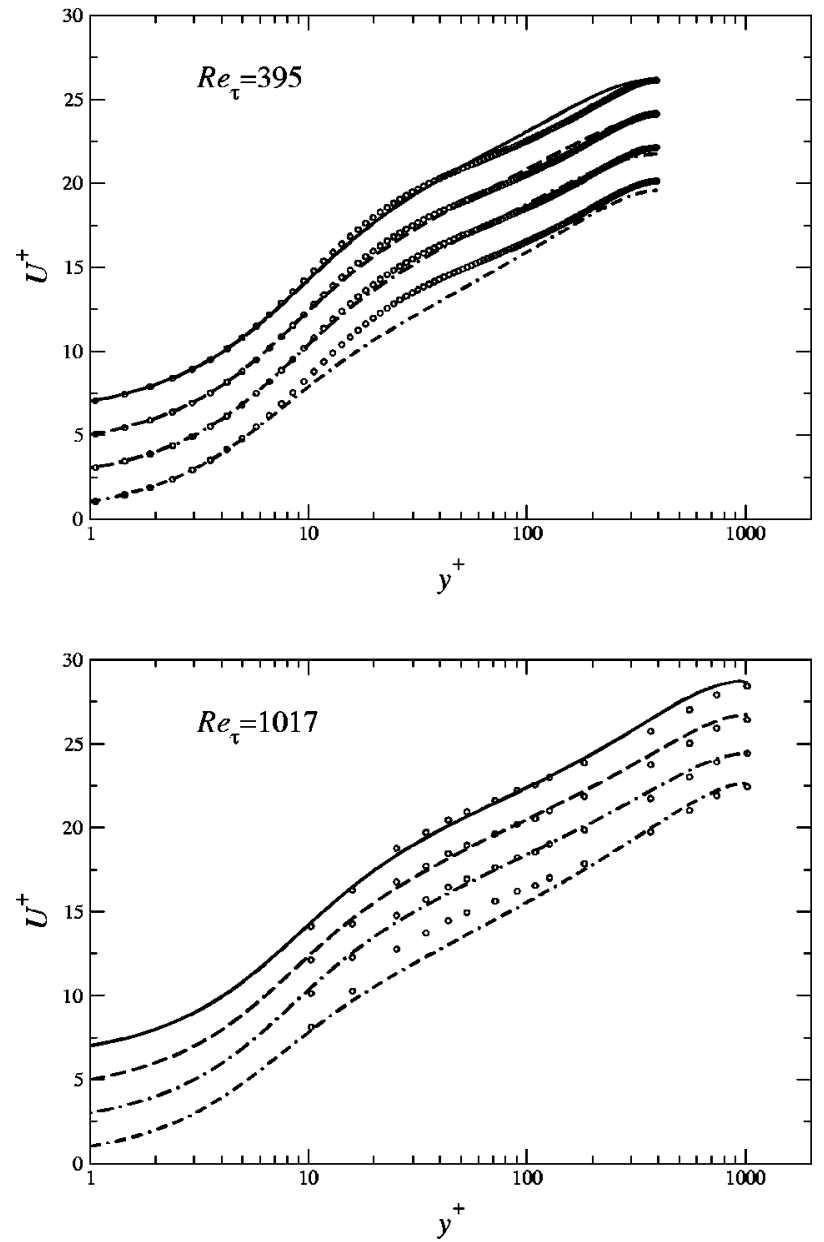

FIG. 7. A comparison of mean velocity profiles from Reynolds stress models with DNS (Ref. 6) and experimental data (Refs. 7, 8) in channel flow. 

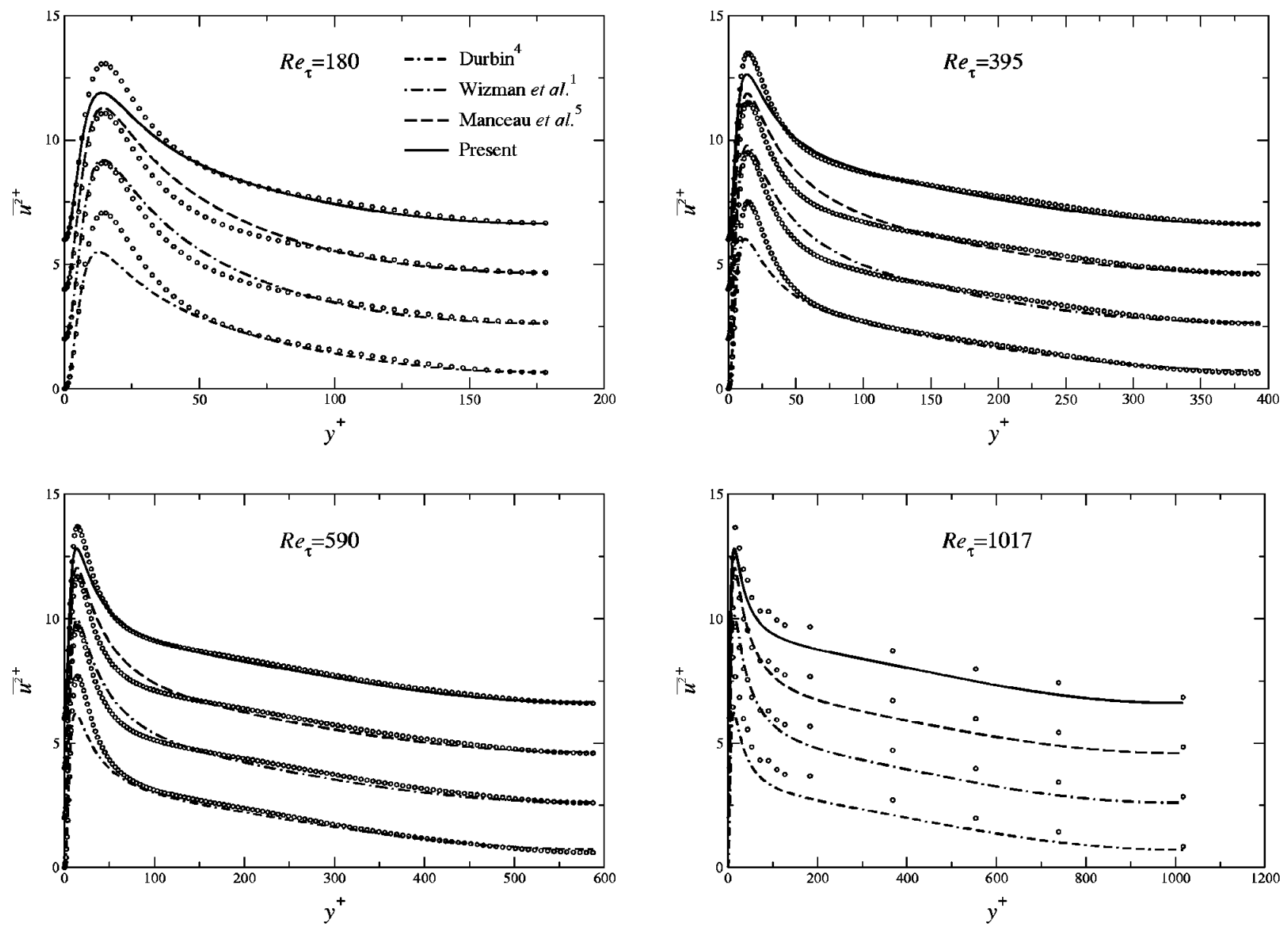

FIG. 8. A comparison of the $\overline{u^{2}+}$ turbulent streamwise stress component from Reynolds stress models with DNS (Ref. 6) and experimental data (Refs. 7, 8) in channel flow.

proportional to the isotropic dissipation rate using $\varepsilon_{i j}^{*}$ $=\left(\overline{u_{i} u_{j}} / K\right) \varepsilon$. The complete set of equations solved for the $\overline{v^{2}}-f$ model, as well as for the full Reynolds stress model, are given in the Appendix. Some results from both the $\overline{v^{2}}$ $-f$ and Reynolds stress forms of the elliptic relaxation procedure compared to both DNS and experimental data will show the improved effect of the new scaling approach.

\section{RESULTS}

Direct simulations of fully developed turbulent channel flow have now become sufficiently complete that such a flow field can be extensively utilized to compare near-wall closure models. There is currently available a consistent set of direct simulation data at $\operatorname{Re}_{\tau}=180,395$, and 590 (see Moser et $a l^{6}{ }^{6}$ ), as well as some experimental results (e.g., Wei and Willmarth ${ }^{7,8}$ at $\operatorname{Re}_{\tau}=1017$ ), that can be used for assessing the performance of this new elliptic relaxation scaling. The eight elliptic relaxation models $\left(\overline{v^{2}}-f\right.$ and Reynolds stress model, four formulations each) are calibrated in order to reproduce as well as possible the four cases from $\operatorname{Re}_{\tau}$ $=180-1017$, while keeping the usual coefficients $\left(C_{\varepsilon 1}\right.$, $C_{\varepsilon 2}$, etc.) as close as possible to their standard values in free shear flows, in order to avoid spoiling the predictions in such flows. The results presented in the following section are thus the best compromise obtainable for each model.
In addition to extending the channel flow assessments to higher values of $\mathrm{Re}_{\tau}$, a further validation of this new rescaling is provided by a comparative assessment of predictions from previous $\overline{v^{2}}-f$ formulations and the present model in flat plate turbulent boundary layer flow. These results are further compared to direct numerical simulation data (see Spalart $^{9}$ 1988) as well as experimental data. ${ }^{10}$

The channel flow computations are performed with a simple finite difference one-dimensional (1-D) code in which the governing equations are normalized by $h$ and $u_{\tau}$, so that $\operatorname{Re}_{\tau}$ can be imposed. The flat plate boundary layer is solved using a finite volume code on a 2-D grid consisting of 440 $\times 100$ cells in the streamwise and wall-normal directions, respectively. The DNS data ${ }^{9}$ at $\operatorname{Re}_{\theta}=300$ are used as inflow conditions.

\section{A. $\overline{v^{2}}-f$ model}

Figure 1 shows the mean velocity predictions using different forms of the elliptic relaxation model developed previously and the present rescaled model compared to DNS $\left(\operatorname{Re}_{\tau}=180,395,590\right)$ and experimental $\left(\operatorname{Re}_{\tau}=1017\right)$ results in channel flow. The present model as well as the Wizman et $a l .{ }^{1}$ and Manceau et al. ${ }^{5}$ models clearly improve the loglayer predictions of the Durbin model ${ }^{3}$ at all the Reynolds numbers. However, the present rescaled model exhibits a 

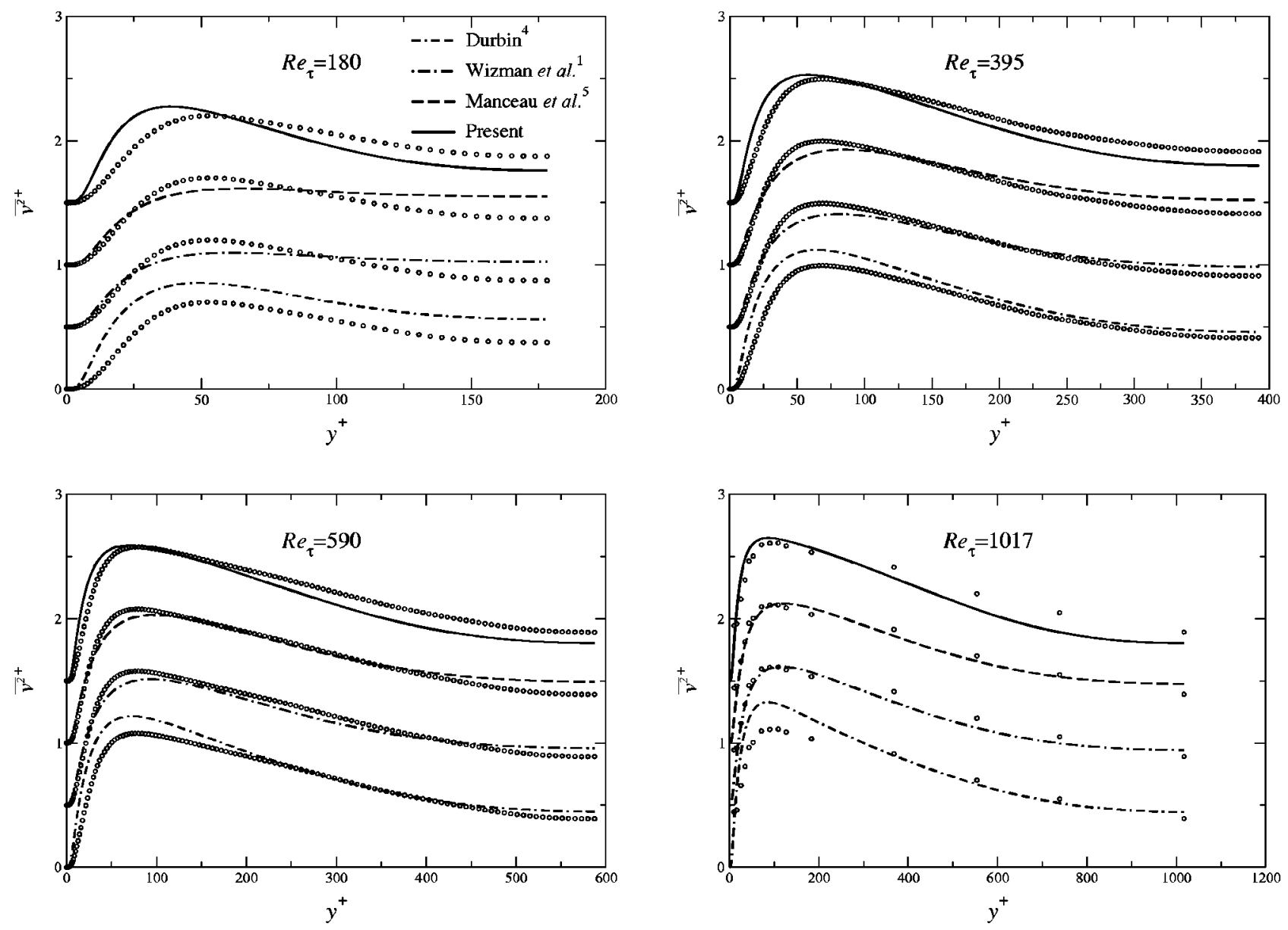

FIG. 9. A comparison of the $\overline{v^{2}}$ turbulent normal stress component from Reynolds stress models with DNS (Ref. 6) and experimental data (Refs. 7, 8) in channel flow.

better sensitivity to reducing the Reynolds number to low values: in particular, the mean velocity profile at $\operatorname{Re}_{\tau}=180$ is much better predicted than with the other models. It must be emphasized that this sensitivity is obtained without introducing any new low-Reynolds number modification in the model.

The corresponding $\overline{v^{2}}$ profiles are shown in Fig. 2. Once again the present model better predicts the entire $y^{+}$distribution (including the peak) compared to the Wizman et al., Manceau et al., and Durbin models over the entire Reynolds number range examined. In the inner layer, all the modified models (including the present model) slightly overpredict, in general, both the DNS and experimental data; however, the original model $\left(\right.$ Durbin $\left.^{3}\right)$ underpredicts the data in this region. In the central region of the flow, a major improvement is obtained with the present model, compared to all the other models, in particular, at the lowest Reynolds number. For the turbulent kinetic energy predictions shown in Fig. 3, all the modified models now underpredict the (DNS) data in the inner region, whereas the original model overpredicts the data and, in particular, the peak of kinetic energy. In addition, the present, rescaled model better predicts the simulation data than the other modified models in the log layer. [Note that for the experimental data, the measurements were obtained from a two-component LDA system that did not mea- sure the $w$ (spanwise) Reynolds stress component. Thus, no kinetic energy data are available.]

Both the mean and turbulent quantities shown suggest that the current, rescaled elliptic relaxation procedure can accurately replicate the flow field over a Reynolds number range. This is further substantiated by examining the skin friction variation over the range of $\operatorname{Re}_{\tau}$ studied. Figure 4 shows the variation of $C_{f}\left(=2 u_{\tau}^{2} / U_{c}^{2}, U_{c}\right.$ centerline velocity) with $\operatorname{Re}_{c}\left(=U_{c} h / \nu\right)$ using the different elliptic relaxation formulations. All the modified formulations better predict the $C_{f}$ variations relative to the original Durbin model. The present rescaled formulation tends to predict better $C_{f}$ levels at the lower Reynolds numbers, but as the value of $\operatorname{Re}_{c}$ increases all the modified models tend to the same levels. Note that even though $U_{c}$ appears in both $C_{f}$ and $\operatorname{Re}_{c}$ (and $u_{\tau}$ is fixed for each case), the $C_{f}$ evolution does not depend on the computational approach. For example, by imposing the centerline velocity (i.e., $\operatorname{Re}_{c}$ ) and evaluating the wall shear stress from the results, exactly the same $C_{f}$ behavior is obtained.

While the results shown here for the $\overline{v^{2}}-f$ model support the analysis of the previous section, which showed that the new scaling neutralizes the adverse effect in the log layer, the present model was also found to give improved overall 

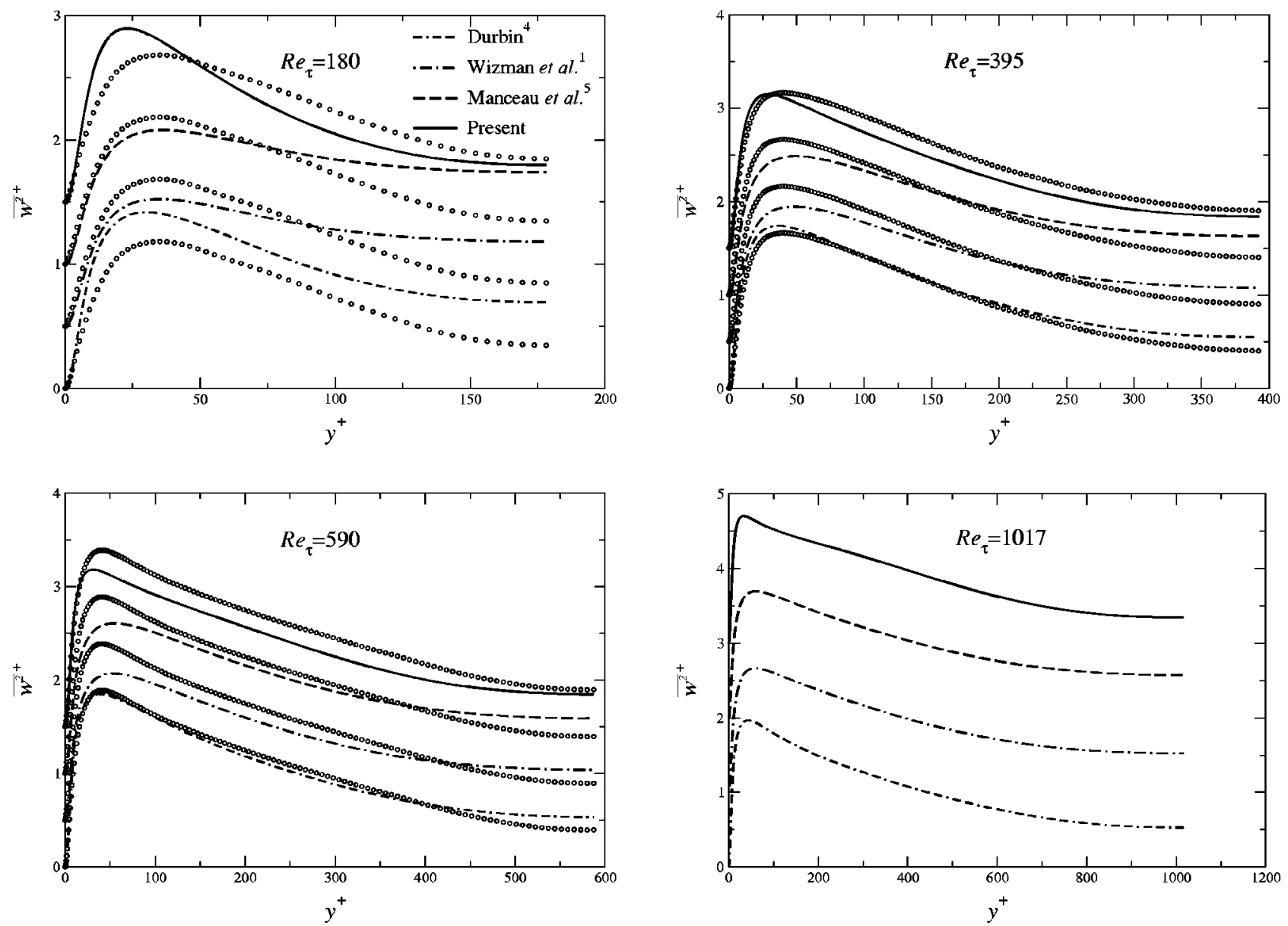

FIG. 10. A comparison of $\overline{w^{2}}$ turbulent spanwise stress component from Reynolds stress models with DNS (Ref. 6) and experimental data (Refs. 7, 8) in channel flow.

predictions across the channel relative to not only the Durbin model but the other modified models. Before proceeding onto a channel flow analysis of a Reynolds stress model based on the rescaled elliptic relaxation formulation, it is of interest to examine the performance of the rescaled $\overline{v^{2}}-f$ model in a flat plate turbulent boundary layer flow.

As was shown in Fig. 4 for the channel flow $C_{f}$ comparisons, the rescaled model displayed the proper Reynolds number dependency over a range of Reynolds numbers. The same trend can also be seen in Fig. 5, where the $C_{f}$ predictions as a function of momentum thickness Reynolds number $\operatorname{Re}_{\theta}$ are compared with both the DNS ${ }^{9}$ and experimental ${ }^{10}$ data. The rescaled $\overline{v^{2}}-f$ model closely predicts the lower $\operatorname{Re}_{\theta}$ DNS data and is asymptotically consistent with the higher $\operatorname{Re}_{\theta}$ experimental data. In addition, the behavior is qualitatively similar to the result obtained from the von Kàrmàn-Schoenherr correlation given here in the form ${ }^{20}$

$$
\frac{1}{C_{f}}=17.08\left(\log \operatorname{Re}_{\theta}\right)^{2}+25.11\left(\log \operatorname{Re}_{\theta}\right)+6.012 .
$$

Figure 5 also shows significant discrepancies between two previous forms of the $\overline{v^{2}}-f$ model. The original $\overline{v^{2}}-f$ version in the figure was used by Durbin $^{21}$ to compute the boundary layer and uses the production-to-dissipation rate ratio $P / \varepsilon$ in the variable coefficient $C_{\varepsilon 1}$. The stand- ard $\overline{v^{2}}-f$ version in Fig. 5 was introduced by Parneix, Durbin, and Behnia ${ }^{17}$ and replaces $P / \varepsilon$ by $\sqrt{K / \overline{v^{2}}}$ in $C_{\varepsilon 1}$ (this version is now widely used and is described of the Appendix; it is the version used for the computations of the channel flow in the present study). As Fig. 5 shows, the standard version does not reproduce the variation of the friction coefficient with Reynolds number very well. In contrast, the original version reproduces the trend at high Reynolds number very well, but not at low Reynolds number. Only the present rescaled model is able to predict the correct evolution over the whole range of Reynolds numbers examined $\left(\operatorname{Re}_{\theta}\right.$ ranges from 670 to 13052 , which corresponds to $\mathrm{Re}_{\delta}$ from 5200 to 120000 , where $\delta$ is the boundary layer thickness, and, in wall units, to $\operatorname{Re}_{\delta+}$ from 250 to 4100 ).

The boundary-layer mean velocity profiles at $\operatorname{Re}_{\theta}=670$ and 13052 are shown in Fig. 6. These two Reynolds numbers delimit the range covered by the DNS and experimental data. At $\operatorname{Re}_{\theta}=670$, the current rescaled model gives the better overall prediction of the mean velocity profiles compared to the other two versions. At $\operatorname{Re}_{\theta}=13052$, all the predictions deteriorate relative to the lower Reynolds number. The rescaled model continues to outperform the standard version, but the predictions of the original model have improved and are as accurate as those of the rescaled model. 

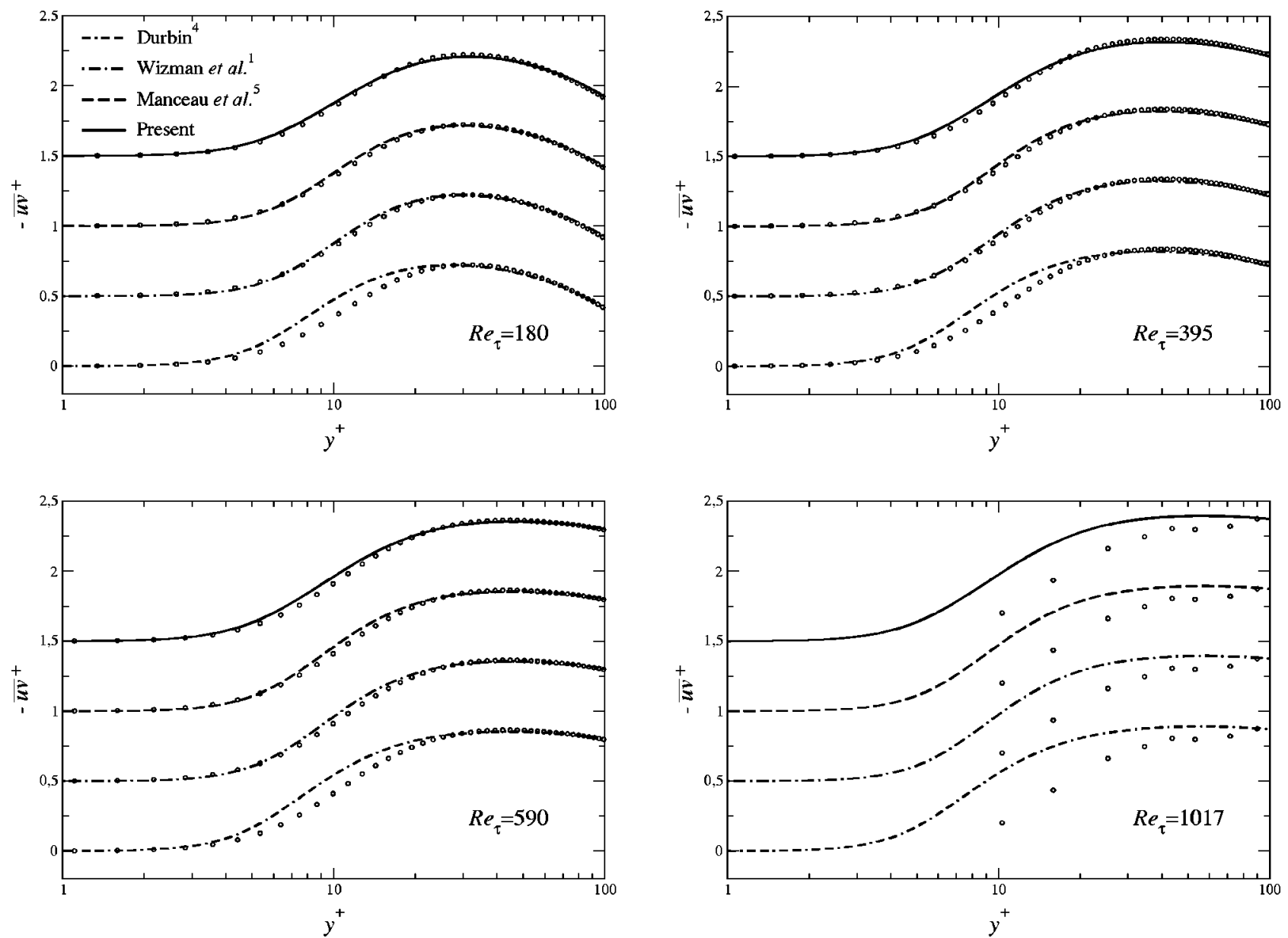

FIG. 11. A comparison of turbulent shear stress profiles from Reynolds stress models with DNS (Ref. 6) and experimental data (Refs. 7, 8) in channel flow.

\section{B. Reynolds stress model}

The elliptic relaxation form of the Reynolds stress model has not received as much attention and validation as the $\overline{v^{2}}$ $-f$ form. Nevertheless, it is of interest to examine the effect of this isotropic rescaling on a closure model that best describes the anisotropies of the turbulence.

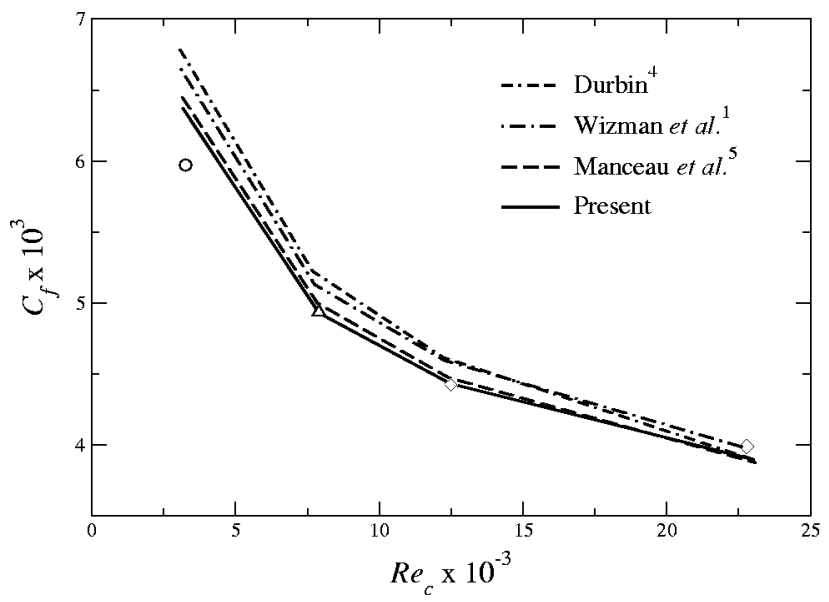

FIG. 12. The variation of the skin friction coefficient over the Reynolds number range for Reynolds stress models in channel flow: $\bigcirc, \operatorname{Re}_{c}=3260$ $\left(\operatorname{Re}_{\tau}=180\right) ; \square, \operatorname{Re}_{c}=7897\left(\operatorname{Re}_{\tau}=395\right) ; \Delta, \operatorname{Re}_{c}=12485\left(\operatorname{Re}_{\tau}=590\right) ; \diamond$, $\operatorname{Re}_{c}=22776\left(\operatorname{Re}_{\tau}=1017\right)$.
Figure 7 shows the mean velocity profiles computed using the four different elliptic relaxation procedures. The three modified formulations are able to predict the correct velocity distributions across the channel with the Manceau et al. ${ }^{2}$ form providing the best predictions on balance across the Reynolds number range. Similar to the $\overline{v^{2}}-f$ results just presented, the new rescaled formulation is more sensitive to the reduction of Reynolds number; although Fig. 7 shows that for the case $\operatorname{Re}_{\tau}=180$, the formulations of Wizman et al. and Manceau et al. also give good predictions.

The distribution of the normal stress components are shown in Figs. 8-10. For the $\overline{u^{2}}$ component (Fig. 8), all the profiles are well predicted in the log layer; although only the Wizman et al. and Manceau et al. formulations are able to reproduce the peak value of $\overline{u^{2}}$. The $\overline{v^{2}}$ normal stress distributions are shown in Fig. 9. Overall, the agreement is not as good as the $\overline{u^{2}}$ component, particularly in the outer layer regions as the centerline is approached. It also appears that the formulations of Wizman et al. and Manceau et al. are more accurate in the near-wall region $\left(y^{+}<30\right)$ for all the Reynolds numbers, while the present rescaled model gives better results in the center of the channel at low Reynolds number. Very similar conclusions can be drawn for $\overline{w^{2}}$ profiles shown in Fig. 10, except that the original formulation predicts a better (higher) peak of $\overline{w^{2}}$ for the cases $\mathrm{Re}_{\tau}$ $\geqslant 395$. [Recall that for the experimental data, the $w$ (span- 
TABLE I. Elliptic relaxation operator and coefficients used for the different formulations of the $\overline{v^{2}}-f$ model.

\begin{tabular}{|c|c|c|c|c|c|c|c|c|c|c|c|c|}
\hline Model & $\mathcal{L} f$ & $C_{\varepsilon 1}$ & $C_{\varepsilon 2}$ & $C_{\mu}$ & $\sigma_{\varepsilon}$ & $\sigma_{K}$ & $C_{L}$ & $C_{\eta}$ & $C_{T}$ & $A_{1}$ & $C_{1}$ & $C_{2}$ \\
\hline Durbin (Ref. 17) & $f-L^{2} \nabla^{2} f$ & 1.4 & 1.9 & 0.22 & 1.3 & 1 & 0.25 & 85 & 6 & 0.045 & 1.4 & 0.45 \\
\hline Wizman et al. (Ref. 1) & $f-\nabla^{2}\left(L^{2} f\right)$ & 1.44 & 1.91 & 0.22 & 1.3 & 1 & 0.35 & 65 & 6 & 0.05 & 1.8 & 0.6 \\
\hline Manceau et al. (Ref. 5) & $\begin{array}{c}{\left[1+16 \beta(\nabla L)^{2}\right] f} \\
-L^{2} \nabla^{2} f-8 \beta L \nabla L \cdot \nabla f\end{array}$ & 1.44 & 1.91 & 0.22 & 1.3 & 1 & 0.275 & 85 & 6 & 0.045 & 1.8 & 0.6 \\
\hline Present & $f-L^{2} \nabla^{2} f$ & 1.44 & 1.91 & 0.22 & 1.3 & 1 & 0.38 & 85 & 6 & 0.045 & 1.8 & 0.6 \\
\hline
\end{tabular}

wise) Reynolds stress component was not measured.]

For fully developed channel flow, the turbulent shear stress is the only Reynolds stress component that directly affects the streamwise momentum equation. As Fig. 11 shows, all the modified models are able to closely predict the behavior of the shear stress through the buffer layer over the range of $\operatorname{Re}_{\tau}$ covered by the DNS data, whereas the original model overpredicts $\overline{u v}$ for $y^{+} \leqslant 20$. The experimental data, however, are not well reproduced by any of the models over the range of $y^{+}$values shown. For larger values of $y^{+}$(not shown) the predictions are in very good agreement with both the DNS and experimental data.

Finally, as was shown with the $\overline{v^{2}}-f$ results for the skin friction coefficient, the elliptic relaxation procedure correctly sensitizes the predictions to Reynolds number variations over the range studied. This also holds for the Reynolds stress models, as shown in Fig. 12. The figure shows that all the models correctly predict the qualitative Reynolds number variation, and that the present, rescaled model more closely replicates the quantitative variation.

\section{CONCLUSIONS}

The examples discussed here have shown that the present, rescaled elliptic relaxation formulation is a viable alternative to previous modified elliptic relaxation formulations that can be used successfully in accurately predicting both the near-wall and log-layer dynamics in both channel flows and boundary layers. Based on the channel flow comparisons at the four Reynolds numbers $\left(\operatorname{Re}_{\tau}=180,395,590\right.$, and 1017) analyzed, the important point emerging from the results is the improvement of the predictions in the center of the channel due to the rescaling by $\varepsilon K$ instead of $K$ alone, as well as the comparable performance to the other (modified) models over the remainder of the channel. The better sensitivity to the Reynolds number reduction also observed is probably a consequence of this improvement, insofar as, at low Reynolds number, a coupling between the near-wall and outer layer regions of the flow appears. This leads to a sig- nificantly better prediction of the $C_{f}$ number variations, particularly when compared to the original formulation of Durbin. $^{3}$

The boundary layer comparisons revealed that previous versions ${ }^{17,21}$ of the $\overline{v^{2}}-f$ model were not able to as accurately compute such flows as the new rescaled model. Both skin friction variation over a broad range of Reynolds numbers and corresponding mean velocity profiles at the low and high Reynolds number limits examined quantified the differences between models. This analysis also led to the conclusion that the original version ${ }^{21}$ is more accurate in the boundary-layer predictions than the current standard model. ${ }^{17}$

The proposed dissipation rate modification is a simple and efficient way to suppress a major negative side effect of the original model in the log layer. It is based on the intuitive hypothesis that the natural scaling by $\varepsilon$ must be introduced in the process, contrary to the Manceau et al. ${ }^{5}$ model that is based on a refinement of the shape assumption for the twopoint correlations involved in the redistribution term under its integral form.

The simplicity of the modification to the original models $\left(\overline{v^{2}}-f\right.$ and Reynolds stress model), compared to the gain in accuracy in the log-layer profiles and the friction coefficient, makes the new proposal attractive. Indeed, considering the successes achieved previously using only the original turbulent kinetic energy scaling, the results presented here do suggest that extensions to more complex flows will also yield improved overall predictions in the near-wall region for both mean and turbulent quantities. Such more complex flows will be the subject of future studies.

\section{APPENDIX: RESCALED $\overline{v^{2}}-f$ AND REYNOLDS STRESS MODELS}

1. $\overline{v^{2}}-f$

The $\overline{v^{2}}-f$ model consists of the transport equations for the turbulent kinetic energy, the isotropic dissipation rate, and the $\overline{v^{2}}\left(=\tau_{22}\right)$ normal stress component,

TABLE II. Coefficients used for the different formulations of the RSM model. For the form of the elliptic relaxation operator $\mathcal{L}$, see Table I.

\begin{tabular}{|c|c|c|c|c|c|c|c|c|c|c|c|c|c|c|c|c|}
\hline Model & $C_{\varepsilon 1}$ & $C_{\varepsilon 2}$ & $C_{\mu}$ & $\sigma_{\varepsilon}$ & $\sigma_{K}$ & $C_{L}$ & $C_{\eta}$ & $C_{T}$ & $A_{2}$ & $C_{1}$ & $C_{1}^{*}$ & $C_{2}$ & $C_{3}$ & $C_{3}^{*}$ & $C_{4}$ & $C_{5}$ \\
\hline Durbin (Ref. 4) & 1.35 & 1.83 & 0.26 & 1.4 & 1 & 0.16 & 80 & 6 & 0.1 & 3.4 & 1.8 & 4.2 & 0.8 & 1.3 & 1.25 & 0.4 \\
\hline Wizman et al. (Ref. 1) & 1.35 & 1.83 & 0.26 & 1.4 & 1 & 0.29 & 80 & 6 & 0.1 & 3.4 & 1.8 & 4.2 & 0.8 & 1.3 & 1.25 & 0.4 \\
\hline Manceau et al. (Ref. 5) & 1.35 & 1.83 & 0.26 & 1.4 & 1 & 0.28 & 80 & 6 & 0.1 & 3.4 & 1.8 & 4.2 & 0.8 & 1.3 & 1.25 & 0.4 \\
\hline Present & 1.35 & 1.83 & 0.26 & 1.4 & 1 & 0.23 & 80 & 6 & 0.12 & 3.4 & 1.8 & 4.2 & 0.8 & 1.15 & 1.25 & 0.4 \\
\hline
\end{tabular}




$$
\begin{aligned}
& \frac{D K}{D t}=\mathcal{P}-\varepsilon+\frac{\partial}{\partial x_{j}}\left[\left(\nu+\frac{\nu_{t}}{\sigma_{K}}\right) \frac{\partial K}{\partial x_{j}}\right], \\
& \frac{D \varepsilon}{D t}=\frac{1}{T}\left(C_{\varepsilon 1}^{\prime} \mathcal{P}-C_{\varepsilon 2} \varepsilon\right)+\frac{\partial}{\partial x_{j}}\left[\left(\nu+\frac{\nu_{t}}{\sigma_{\varepsilon}}\right) \frac{\partial \varepsilon}{\partial x_{j}}\right], \\
& \frac{D \overline{v^{2}}}{D t}=\varepsilon K f-\frac{\overline{v^{2}}}{K} \varepsilon+\frac{\partial}{\partial x_{j}}\left[\left(\nu+\frac{\nu_{t}}{\sigma_{K}}\right) \frac{\partial \overline{v^{2}}}{\partial x_{j}}\right],
\end{aligned}
$$

where $\nu_{t}=C_{\mu} \overline{v^{2}} T$. An elliptic relaxation equation is solved for the function $f$ that models the redistributive effect in the $\overline{v^{2}}$ equation, and is identical to the equation satisfied by the wall normal component $f_{22}$ of the full Reynolds stress model in a channel flow,

$$
\mathcal{L} f=\frac{1}{\varepsilon K}\left(\Pi_{22}+2 \varepsilon b_{22}\right) .
$$

Using the Launder, Reece, and Rodi ${ }^{11}$ model for $\Pi_{i j}$, and replacing $\varepsilon / K$ by $1 / T$ to avoid a singularity at the wall, leads to

$$
\mathcal{L} f=\left(C_{1}-1\right) \frac{2 / 3-\overline{v^{2}} / K}{\varepsilon T}+\frac{2}{3} C_{2} \frac{P}{\varepsilon K},
$$

where $\mathcal{P}=2 K b_{k l} S_{l k}\left(=2 \nu_{t} S_{k l} S_{l k}\right)$, with

$$
\begin{aligned}
& b_{i j}=\left(\frac{\tau_{i j}}{2 K}-\frac{\delta_{i j}}{3}\right), \quad T=\max \left(\frac{K}{\varepsilon}, C_{T} \sqrt{\frac{\nu}{\varepsilon}}\right), \\
& L=C_{L} \max \left[\frac{K^{3 / 2}}{\varepsilon}, C_{\eta}\left(\frac{\nu^{3}}{\varepsilon}\right)^{1 / 4}\right], \\
& C_{\varepsilon 1}^{\prime}=C_{\varepsilon 1}\left[1+A_{1}\left(\frac{K}{\overline{v^{2}}}\right)^{1 / 2}\right] .
\end{aligned}
$$

These equations are then solved subject to the boundary conditions at the solid boundary,

$$
K=0, \quad \varepsilon=\frac{2 \nu K_{(1)}}{y_{(1)}^{2}}, \quad \overline{v^{2}}=0, \quad f=-\frac{20 \nu^{2} \bar{v}_{(1)}^{2}}{\varepsilon^{2} y_{(1)}^{4}},
$$

where the subscript (1) denotes the value at the first interior computational grid point away from the wall.

Recall that the other formulations applied in this study ${ }^{1,3,5}$ use a different scaling. The set of coefficients used with the different models and the form of the operator $\mathcal{L}$ are given in Table I.

\section{Reynolds stress model}

The transport equation for the Reynolds stress $\tau_{i j}\left(=\overline{u_{i} u_{j}}\right)$ is given by

$$
\begin{aligned}
\frac{D \tau_{i j}}{D t}= & -\tau_{i k} \frac{\partial U_{j}}{\partial x_{k}}-\tau_{j k} \frac{\partial U_{i}}{\partial x_{k}}+\varepsilon K f_{i j}-\frac{\tau_{i j}}{K} \varepsilon \\
& +\frac{\partial}{\partial x_{l}}\left(\frac{C_{\mu}}{\sigma_{K}} \tau_{l m} T \frac{\partial \tau_{i j}}{\partial x_{m}}\right)+\nu \frac{\partial^{2} \tau_{i j}}{\partial x_{l} \partial x_{l}},
\end{aligned}
$$

and for the dissipation rate by

$$
\frac{D \varepsilon}{D t}=\frac{1}{T}\left(C_{\varepsilon 1}^{\prime} \mathcal{P}-C_{\varepsilon 2} \varepsilon\right)+\frac{\partial}{\partial x_{l}}\left(\frac{C_{\mu}}{\sigma_{\varepsilon}} \tau_{l m} T \frac{\partial \varepsilon}{\partial x_{m}}\right),
$$

where $\mathcal{P}=2 K b_{k l} S_{l k}$. The elliptic relaxation equation for $f_{i j}$ that models the redistributive effect in the Reynolds stress transport equations is

$$
\mathcal{L} f_{i j}=\frac{1}{\varepsilon K}\left(\Pi_{i j}+2 \varepsilon b_{i j}\right) \text {. }
$$

The SSG model ${ }^{13}$ is used for $\Pi_{i j}$, and $\varepsilon / K$ is replaced by $1 / T$ to avoid a singularity at the wall. This yields

$$
\begin{aligned}
\mathcal{L} f_{i j}= & -\left(\left(C_{1}-2\right) \frac{1}{\varepsilon T}+C_{1}^{*} \frac{\mathcal{P}}{\varepsilon K}\right) b_{i j}+C_{2} \frac{1}{\varepsilon T} \operatorname{dev}\left(b_{i k} b_{k j}\right) \\
& +\left[C_{3}-C_{3}^{*}\left(b_{l m} b_{m l}\right)^{1 / 2}\right] \frac{S_{i j}}{\varepsilon}+\frac{C_{4}}{\varepsilon} \operatorname{dev}\left(b_{i k} S_{k j}\right. \\
& \left.+S_{i k} b_{k j}\right)+\frac{C_{5}}{\varepsilon}\left(b_{i k} W_{k j}-W_{i k} b_{k j}\right),
\end{aligned}
$$

with

$$
\begin{aligned}
& T=\max \left(\frac{K}{\varepsilon}, C_{T} \sqrt{\frac{\nu}{\varepsilon}}\right), \quad L=C_{L} \max \left[\frac{K^{3 / 2}}{\varepsilon}, C_{\eta}\left(\frac{\nu^{3}}{\varepsilon}\right)_{(\mathrm{A} 13)}^{1 / 4}\right], \\
& C_{\varepsilon 1}^{\prime}=C_{\varepsilon 1}\left(1+A_{2} \frac{\mathcal{P}}{\varepsilon}\right) .
\end{aligned}
$$

These equations are then solved subject to the boundary conditions at the solid boundary:

$$
\begin{aligned}
& \tau_{i j}=0, \quad \varepsilon=\frac{2 \nu K_{(1)}}{y_{(1)}^{2}}, \\
& f_{22}=-\frac{20 \nu^{2} \bar{v}_{(1)}^{2}}{\varepsilon^{2} y_{(1)}^{4}}, \quad f_{12}=-\frac{20 \nu^{2} \overline{u v}_{(1)}}{\varepsilon^{2} y_{(1)}^{4}}, \\
& f_{11}=f_{33}=-\frac{1}{2} f_{22},
\end{aligned}
$$

where the subscript (1) denotes the value at the first interior computational grid point away from the wall.

As with the $\overline{v^{2}}-f$ model, the form of the operator $\mathcal{L}$ depends on the choice of either the Durbin, ${ }^{3}$ Wizman et al. ${ }^{1}$ Manceau et al., ${ }^{2}$ or present formulations (see Table I). The coefficients used with the different elliptic relaxation formulations are given in Table II.

${ }^{1}$ V. Wizman, D. Laurence, M. Kanniche, P. Durbin, and A. Demuren, "Modeling near-wall effects in second-moment closures by elliptic relaxation,' Int. J. Heat Fluid Flow 17, 255 (1996).

${ }^{2}$ R. Manceau and K. Hanjalic, "A new form of the elliptic relaxation equation to account for wall effects in RANS modeling," Phys. Fluids 12, 2345 (2000).

${ }^{3}$ P. A. Durbin, "Near-wall turbulence closure modeling without 'damping functions,' "' Theor. Comput. Fluid Dyn. 3, 1 (1991).

${ }^{4}$ P. A. Durbin, “A Reynolds stress model for near-wall turbulence," J. Fluid Mech. 249, 465 (1993).

${ }^{5}$ R. Manceau, M. Wang, and D. Laurence, "Inhomogeneity and anisotropy effects on the redistribution term in Reynolds-averaged Navier-Stokes modelling," J. Fluid Mech. 438, 307 (2001).

${ }^{6}$ R. D. Moser, J. Kim, and N. N. Mansour, "Direct numerical simulation of turbulent channel flow up to $\mathrm{Re}_{\tau}=590$," Phys. Fluids 11, 943 (1999).

${ }^{7}$ T. Wei and W. W. Willmarth, "Reynolds-number effects on the structure of a turbulent channel flow," J. Fluid Mech. 204, 57 (1989).

${ }^{8}$ T. Wei and W. W. Willmarth, "PCH12: Fully developed turbulent channel 
flow experiments," in A Selection of Test Cases for the Validation of Large-Eddy Simulations of Turbulent Flows, AGARD AR 345, 1998.

${ }^{9}$ P. Spalart, "Direct simulation of a turbulent boundary layer up to $\operatorname{Re}_{\theta}$ $=1410$," J. Fluid Mech. 187, 61 (1988).

${ }^{10}$ D. R. Smith and A. J. Smits, "TBL00: Flat plate, zero-pressure-gradient TBL," in A Selection of Test Cases for the Validation of Large-Eddy Simulations of Turbulent Flows, AGARD AR 3451998.

${ }^{11}$ B. E. Launder, G. Reece, and W. Rodi, "Progress in the development of a Reynolds stress turbulence closure,” J. Fluid Mech. 68, 537 (1975).

${ }^{12}$ T. J. Craft and B. E. Launder, "Computation of impinging flows using second-moment closures," 8th Symposium on Turbulent Shear Flows, Munich, Germany, 1991, Vol. 8.

${ }^{13}$ C. G. Speziale, S. Sarkar, and T. B. Gatski, "Modeling the pressure-strain correlation of turbulence: an invariant dynamical systems approach," J. Fluid Mech. 227, 245 (1991).

${ }^{14} \mathrm{R}$. Manceau, "Reproducing the blocking effect of the wall in one-point turbulence models," European Congress on Computational Methods of Applied Sciences and Engineering, Barcelona, Spain, 2000.

${ }^{15}$ D. Laurence and P. Durbin, "Modeling near wall effects in second mo- ment closures by elliptic relaxation," Proceedings of the Summer Program, CTR, Stanford University/NASA Ames Research Center, 1994.

${ }^{16}$ P. A. Durbin and D. Laurence, "Nonlocal effects in single point closure," Turbulence Research Associates, 96 Meeting, Seoul, Korea, 1996.

${ }^{17}$ S. Parneix, P. A. Durbin, and M. Behnia, "Computation of 3-D turbulent boundary layers using the $\overline{v^{2}}-f$ model," Flow, Turbul. Combust. 60, 19 (1998).

${ }^{18}$ R. Manceau, S. Parneix, and D. Laurence, "Turbulent heat transfer predictions using the $\overline{v^{2}}-f$ model on unstructured meshes, " Int. J. Heat Fluid Flow 21, 320 (2000).

${ }^{19}$ F.-S. Lien and G. Kalitzin, "Computations of transonic flow with the $\overline{v^{2}}$ $-f$ turbulence model," Int. J. Heat Fluid Flow 22, 53 (2001).

${ }^{20}$ E. J. Hopkins and M. Inouye, "An evaluation of theories for predicting turbulent skin friction and heat transfer on flat plates at supersonic and hypersonic Mach numbers," AIAA J. 9, 993 (1971).

${ }^{21}$ P. Durbin, "Application of a near-wall turbulence model to boundary layers and heat transfer," Int. J. Heat Fluid Flow 14, 316 (1993). 